The University of San Francisco

USF Scholarship: a digital repository @ Gleeson Library |

Geschke Center

2011

\title{
Electron Density Measurements in a Pulse- Repetitive Microwave Discharge in Air
}

Milka Nikolic

University of San Francisco, mnikolic@usfca.edu

S. Popović

L. Vušković

G. C. Herring

R.J. Exton

Follow this and additional works at: http://repository.usfca.edu/phys

Part of the Physics Commons

\section{Recommended Citation}

Nikolić, M., Popović, S., Vušković, L., Herring, G.C., Exton, R.J. Electron density measurements in a pulse-repetitive microwave discharge in air (2011) Journal of Applied Physics, 110 (11), art. no. 113304. http://dx.doi.org/10.1063/1.3665195

This Article is brought to you for free and open access by the College of Arts and Sciences at USF Scholarship: a digital repository @ Gleeson Library | Geschke Center. It has been accepted for inclusion in Physics and Astronomy by an authorized administrator of USF Scholarship: a digital repository @ 


\section{Electron density measurements in a pulse-repetitive microwave discharge in air}

M. Nikolić ', S. Popović' L. Vušković' G. C. Herring, and R. J. Exton

Citation: Journal of Applied Physics 110, 113304 (2011); doi: 10.1063/1.3665195

View online: http://dx.doi.org/10.1063/1.3665195

View Table of Contents: http://aip.scitation.org/toc/jap/110/11

Published by the American Institute of Physics 


\title{
Electron density measurements in a pulse-repetitive microwave discharge in air
}

\author{
M. Nikolić, ${ }^{1, a)}$ S. Popović, ${ }^{1}$ L. Vušković, ${ }^{1}$ G. C. Herring, ${ }^{2}$ and R. J. Exton ${ }^{2}$ \\ ${ }^{1}$ Department of Physics, Center for Accelerator Science, Old Dominion University, Norfolk, \\ Virginia 23529, USA \\ ${ }^{2}$ NASA Langley Research Center, Hampton, Virginia 23681, USA
}

(Received 19 June 2011; accepted 28 October 2011; published online 7 December 2011)

\begin{abstract}
We have developed a technique for absolute measurements of electron density in pulse-repetitive microwave discharges in air. The technique is based on the time-resolved absolute intensity of a nitrogen spectral band belonging to the Second Positive System, the kinetic model and the detailed particle balance of the $N_{2} C^{3} \Pi_{u}(v=0)$ state. This new approach bridges the gap between two existing electron density measurement methods (Langmuir probe and Stark broadening). The electron density is obtained from the time-dependent rate equation for the population of $N_{2} C^{3} \Pi_{u}$ $(v=0)$ using recorded waveforms of the absolute $C^{3} \Pi_{u} \rightarrow B^{3} \Pi_{g}(0-0)$ band intensity, the forward and reflected microwave power density. Measured electron density waveforms using numerical and approximated analytical methods are presented for the case of pulse repetitive planar surface microwave discharge at the aperture of a horn antenna covered with alumina ceramic plate. The discharge was generated in air at 11.8 Torr with a X-band microwave generator using $3.5 \mu \mathrm{s}$ microwave pulses at peak power of $210 \mathrm{~kW}$. In this case, we were able to time resolve the electron density within a single $3.5 \mu$ s pulse. We obtained $(9.0 \pm 0.6) \times 10^{13} \mathrm{~cm}^{-3}$ for the peak and $(5.0 \pm 0.6) \times 10^{13} \mathrm{~cm}^{-3}$ for the pulse-average electron density. The technique presents a convenient, non-intrusive diagnostic method for local, time-defined measurements of electron density in short duration discharges near atmospheric pressures. (C) 2011 American Institute of Physics. [doi:10.1063/1.3665195]
\end{abstract}

\section{INTRODUCTION}

A simple and accurate technique for acquiring quantitative information on the spatial-temporal variation of electron density in pulse-repetitive discharges at or near atmospheric pressure is presently needed. Electron density affects the energy balance and heat transfer, kinetics, and global properties of the plasma, such as wave transmission reflectivity, absorption, and scattering. ${ }^{1}$ Most plasmas of interest are non-equilibrium, non-stationary, fast moving, and chemically reactive where it can be very challenging to determine the time-resolved electron density distributions. This is especially the case of pulse-repetitive discharges near atmospheric pressures. Conventional diagnostic techniques, such as Langmuir probes, are applicable for plasmas with densities up to $10^{11}-10^{12} \mathrm{~cm}^{-3}$ at least an order of magnitude lower than needed in air discharges at high pressures. ${ }^{2}$ The most commonly used non-intrusive technique is based on the Stark broadening of hydrogen Balmer lines, but this technique has been applicable with reasonable accuracy for highdensity plasmas $\left(>10^{14} \mathrm{~cm}^{-3}\right)$. Thomson scattering can also be applied as a diagnostic tool in determining plasma parameters, but for this technique, we need to employ high intensity laser radiation, which may change the structure of plasma. ${ }^{3}$ The use of microwave absorption, interferometry and scattering is difficult to implement in the pulsed systems,

\footnotetext{
a) Author to whom correspondence should be addressed. Electronic mail: mniko004@odu.edu.
}

due to low intensity, and long wavelength leading to poor spatial-temporal resolution.

In this work, we explore the possibility to apply a new approach, based on the spectral intensities of certain nitrogen bands. The technique is applicable to the measurement of electron density in air or nitrogen-containing plasmas and is a strong candidate to bridge the density gap between those accessible with Langmuir probe and Stark broadening techniques. In particular, we focus on only the Second Positive System band intensity $C^{3} \Pi_{u} \rightarrow B^{3} \Pi_{g}(0-0)$ at $337.1 \mathrm{~nm}$. Previously, ${ }^{4-9}$ an approach was based on a simplified kinetic model, using two electronically excited levels $\left(C^{3} \Pi_{u}\right.$ and $\left.B^{3} \Pi_{g}\right)$ and one singlet ground state $\left(X^{1} \Sigma^{+}\right)$. Kozlov et al. ${ }^{8,9}$ have published important work on the spatio-temporal spectroscopic diagnostics of a dielectric barrier discharge, based on the relative measurements of electrical field and electron density, using the experimentally detected $\mathrm{C}(0-0)$ and $\mathrm{B}(0-0)$ band intensity waveforms and the dependence of excitation rates on the reduced electric field.

The goal is to expand the above approach to the more realistic cases of (a) non-steady state plasma conditions and (b) conditions where a more detailed particle balance is needed. Pulsed plasma regimes, present in a variety of modern plasma applications, are not compatible with a steady-state analysis. Moreover, fast transitions of the ionization-recombination regimes are carried out through the stepwise vibrational excitation and de-excitation processes, where the cross-population between various vibrational levels 
becomes important enough to account for a non-negligible systematic error in the derived electron density. In the analysis, we have included the absolute vibrational state distribution within $C^{3} \Pi_{u}$ state by performing systematic measurements of vibrational state populations at the conditions where the electron density has to be determined.

Our approach requires solution of the differential equation for the $C^{3} \Pi_{u}(v=0)$ population rate which includes contribution from higher vibrational states. In this analysis, we have developed and compared the analytic and the numeric solutions of the equations in the form of parametric relation between electron density and the absolute $C^{3} \Pi_{u}(v=0)$ population. In this paper, we start with the detailed description of the kinetic model and developed a technique for evaluation of electron density. Further, we describe one of our experiments, the pulse-repetitive microwave discharge at the aperture of a horn antenna, which we have chosen for our case study. Finally, we discuss and compare the results obtained with the steady-state analytical and time-dependent numerical methods.

\section{KINETIC MODEL}

In defining the time-dependent kinetics of the discharge, we start by assuming a simplified model which does not include resonant transitions from the excited states of the carrier gas to $N_{2} C^{3} \Pi_{u}$ state that would increase its population density. Further, ionization and recombination processes are excluded from the model due to their small coefficient rates and low ionization coefficient of the plasma $\left(\leq 10^{-4}\right)$. There are various examples in literature including the work of Kim et $a .^{10}$ and Zhu et al. ${ }^{11}$ that support these assumptions. Acknowledging these restrictions, we can state that nitrogen excited electronic state $\left(C^{3} \Pi_{u}\right)$ is affected mainly by three $\operatorname{processes}^{4,5}$ :

1. electron excitation from the ground state

$$
e+N_{2}\left(X^{1} \Sigma^{+}\right) \rightarrow e+N_{2}\left(C^{3} \Pi_{u}\right)
$$

2. radiative transition

$$
N_{2}\left(C^{3} \Pi_{u}\right) \rightarrow N_{2}\left(B^{3} \Pi_{g}\right)+h \nu
$$

3. quenching (collisional deactivations)

$$
N_{2}\left(C^{3} \Pi_{u}\right)+N_{2}\left(X^{1} \Sigma^{+}\right) \rightarrow 2 N_{2}\left(X^{1} \Sigma^{+}\right) .
$$

Among these transitions, only transitions involving the vibrational ground state of each electronic state are considered.

However, experimental data indicated that some transitions involving levels other than vibrational ground states are significant enough to be included in the kinetic model. They are:

(a) vibrational excitation/deexcitation by electron impact $(e-\mathrm{V})$

$$
e+N_{2}\left(C^{3} \Pi_{u}, 0\right) \rightleftharpoons e+N_{2}\left(C^{3} \Pi_{u}, v\right), \quad v=1,2, \ldots
$$

(b) vibration-vibration collision processes (V-V)

$$
\begin{aligned}
& N_{2}\left(C^{3} \Pi_{u}, 0\right)+N_{2}\left(C^{3} \Pi_{u}, v\right) \rightleftharpoons N_{2}\left(C^{3} \Pi_{u}, 1\right) \\
& \quad+N_{2}\left(C^{3} \Pi_{u}, v-1\right), v=2,3, \ldots
\end{aligned}
$$

The net population rate of the $N_{2}\left(C^{3} \Pi_{u}\right)$ state is given with

$$
\begin{aligned}
\frac{d\left[N_{2}\left(C_{v=0}\right)\right]}{d t}= & k_{e} N_{e} N_{n(v=0)}-\frac{\left[N_{2}\left(C_{v=0}\right)\right]}{\tau_{e}}-k_{c}\left[N_{2}\left(C_{v=0}\right)\right] N_{n} \\
& -\sum_{v} k_{e}^{0, v} N_{2}\left(C_{v=0}\right) N_{e}+\sum_{v} k_{e}^{v, 0} N_{2}\left(C_{v}\right) N_{e} \\
& -\sum_{v} k_{v, v-1}^{0,1} N_{2}\left(C_{v=0}\right) N_{2}\left(C_{v}\right) \\
& +\sum_{v} k_{v-1, v}^{1,0} N_{2}\left(C_{v=1}\right) N_{2}\left(C_{v-1}\right)
\end{aligned}
$$

where $N_{e}$ is time-dependent electron density, $N_{n}$ is the concentration of molecules in $N_{2}\left(X^{1} \Sigma^{+}\right)$ground state, $N_{2}\left(C_{v}\right)$ corresponds to the population of the excited vibrational state of $N_{2}\left(C^{3} \Pi_{u}\right), k_{e}$ is the electron excitation coefficient of the ground state $(v=0), k_{e}^{0, v}$ and $k_{e}^{v, 0}$ are coefficient rates for $e-\mathrm{V}$ (electron-impact vibrational excitation) of the $N_{2}\left(C^{3} \Pi_{u}\right)$ state, $k_{v, v-1}^{0,1}$ and $k_{v-1, v}^{1,0}$ are coefficient rates vibrationvibration collisions $(\mathrm{V}-\mathrm{V}), \tau_{e}$ is the life time of the $N_{2}\left(C^{3} \Pi_{u}\right)$, and $k_{c}$ represents the rate constant for the collisional deexcitation. We have assumed Boltzmann distribution of vibrational state population which we will prove valid using our experimental data

$$
\left[N_{2}\left(C_{v}\right)\right]=\left[N_{2}\left(C_{v=0}\right)\right] e^{-\frac{E_{v 0}}{k_{B} T_{v}}}
$$

where $E_{v 0}$ is transition energy between $N_{2}\left(C_{v}\right)$ state and $\left[N_{2}\left(C_{v=0}\right)\right]$ state, $T_{v}$ is corresponding vibrational temperature and $k_{B}$ is the Boltzmann constant. In what follows, we will demonstrate that both $e-\mathrm{V}$ and $\mathrm{V}-\mathrm{V}$ processes (the last three terms of Eq. (6)) are negligible comparing to the electronic transitions of $N_{2}\left(C^{3} \Pi_{u}\right)$ state (the first four terms of Eq. (6)).

Pancheshnuyi et al. ${ }^{12}$ investigated a detailed kinetic model of nitrogen discharges by including the quenching process between ground state oxygen molecules and $N_{2}\left(C^{3} \Pi_{u}\right)$ state

$$
N_{2}\left(C^{3} \Pi_{u}\right)+O_{2} \rightarrow N_{2}\left(X^{1} \Sigma_{g}^{+}\right)+O+O .
$$

According to Ref. 12, the quenching rate of the $\mathrm{C}$ state in collisions with oxygen molecules is $2.7 \times 10^{-10} \mathrm{~cm}^{3} / \mathrm{s}$, and the quenching rates in the collisions with nitrogen molecules is $(9 \pm 3) \times 10^{-10} \mathrm{~cm}^{3} / \mathrm{s}$. In the extreme case that all oxygen molecules from the air remain in their ground state, unattached, and not dissociated, reaction (8) would contribute by less then $10 \%$ to the overall quenching of the $\mathrm{C}$ state. This contribution is within the error of nitrogen-induced quenching rate, and may be neglected without increasing the error of the final result. 


\section{A. Steady state solution}

We will start with the analytic analysis of the kinetic model, using the approximation that the electron density and $N_{2}\left(C^{3} \Pi_{u}\right)$ population are constant. Assuming that the population of the upper energy level $\left[N_{2}\left(C^{3} \Pi_{u}\right)\right]$ does not change in time and that contributions from vibrational transitions are negligible, we derive the solution for the steady state from

$$
\frac{d\left[N_{2}\left(C_{v=0}\right)\right]}{d t}=0
$$

After combining relations (6) and (9), neglecting the contribution of upper vibrational levels (last four terms in Eq. (6)), we obtained the electron density relation to be

$$
N_{e}=\left[N_{2}\left(C_{v=0}\right)\right] \frac{1+\tau_{e} k_{c} N_{n}}{\tau_{e} k_{e} N_{n}} .
$$

\section{B. Time dependent numerical solution}

Generally, one can solve numerically Eq. (6) for any input power function provided that a waveform for $k_{e}((E / N)(t))$ is established. Hence,

$$
\begin{aligned}
\frac{d\left[N_{2}\left(C_{v=0}\right)\right]}{d t}= & k_{e}(t) N_{n} N_{e}(t)-\frac{\left[N_{2}\left(C_{v=0}\right)\right]}{\tau_{e}} \\
& -k_{c}\left[N_{2}\left(C_{v=0}\right)\right] N_{n} .
\end{aligned}
$$

Substituting

$$
\begin{gathered}
x=\left[N_{2}\left(C_{v=0}\right)\right], \\
N_{n}=\text { const }=A,
\end{gathered}
$$

and

$$
\frac{1}{\tau_{e}}+k_{c} N_{n}=\text { const }=\omega,
$$

we can express the Eq. (11) in a simplified form

$$
\frac{d x}{d t}+\omega x=A k_{e}(t) N_{e}(t)
$$

One can use the waveform for $x$ to establish experimental values for $N_{e}(t)$.

\section{EXPERIMENTAL SET-UP}

The applicability of the present model has been tested in a surface discharge generated at the aperture of a horn antenna with a pulsed microwave source operating in the X-band $(9.5 \mathrm{GHz}, 210 \mathrm{KW})$ spectral range. ${ }^{6}$ The microwave discharge was obtained in air at the pressure of 11.8 Torr. Experiment was conducted in a static plasma cell evacuated to the background air pressure of the order of 100 mTorr. (The detailed description of the microwave plasma experiment was described in Ref. 6). Spectral data were taken both end-on and side-on, as is shown schematically in Fig. 1. This approach was necessary due to the possible existence of

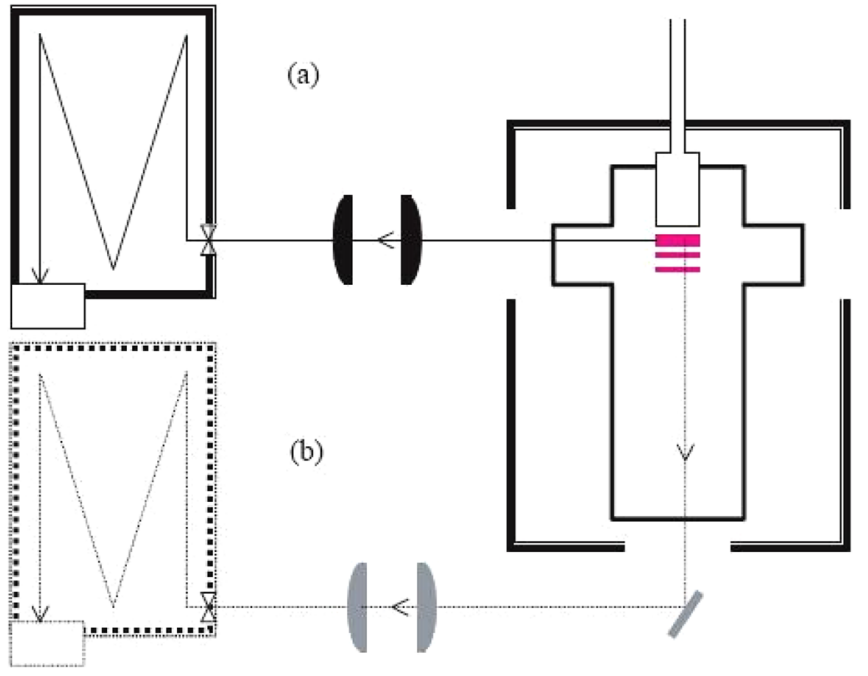

FIG. 1. (Color online) Imaging spectrometer arrangement for (a) side-on and (b) end-on observations.

self-absorption effects when discharge was observed only side-on.

The surface discharge at the aperture of the horn antenna was patterned in bright and dark patches, reflecting the mode structure of the microwave beam. ${ }^{6}$ Due to the mode structure, electric field distribution was undulatory, with maximum to minimum ratio of the order of ten, and four periods covering the E-field side of the horn.

Emission spectra were recorded using a $0.5-\mathrm{m}$ Acton Research Corporation imaging spectrograph connected to the Apogee spectral imaging cameras with Hamamatsu Chargecoupled Device (CCD) and Tektronix CCD detectors. Another camera, from Stanford Computer Optics, Inc., was used to observe the discharge in gated format with time resolution of $50 \mathrm{~ns}$. Alternatively, transient signals at fixed wavelengths were recorded using a photomultiplier tube.

The observed spectra were calibrated using an absolute EG\&G is Edgerton, Germeshausen, and Grier, Inc. black body irradiance source. Calibration graphs of spectral irradiance per count versus wavelength were evaluated for the wavelengths between 250 and $1000 \mathrm{~nm}$ for three spectrometer gratings. We determined the population of particular excited state transitions by using these graphs. Due to low temperature of the calibration source for the spectral range used in present work the statistical error was only $10 \%$.

Time-resolved emission from the microwave discharge plasma was recorded with a photomultiplier tube connected to a digital oscilloscope. Waveforms from 1-3 pulses in a sequence could be recorded on a single oscilloscope data file. A typical time-resolved emission from the $C^{3} \Pi_{u} \rightarrow$ $B^{3} \Pi_{g}(0-0)$ in the transition microwave discharge plasma is given in Fig. 2.

We were also able to record the waveforms of forward and reflected power signal. To calculate the total input power we relied on the nominal peak power of $P_{\text {peak }}=210 \mathrm{~kW}$ and assumed a homogeneous distribution over the horn aperture area. This decision is made because the actual variation of the electric field around the breakdown is relatively small and does not affect the amplitude of the reduced electric 


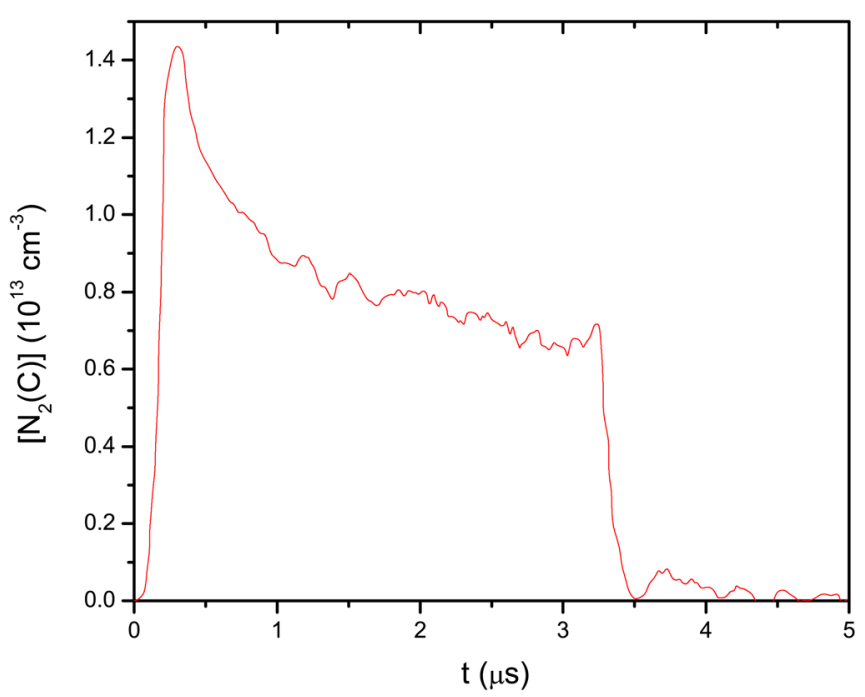

FIG. 2. (Color online) Waveform of the light emission from the $(0-0)$ band of the Second Positive System of Nitrogen.

field. The X-band pyramidal horn $(Z)$ aperture $^{13}$ was $(5.9 \times 7.8) \mathrm{cm}^{2}$. Averaged peak power density at the aperture was therefore

$$
\Pi=\frac{210 \mathrm{~kW}}{5.9 \times 7.8 \mathrm{~cm}^{2}}=4.6 \frac{\mathrm{kW}}{\mathrm{cm}^{2}} .
$$

\section{RESULTS AND DISCUSSION}

\section{A. Microwave power measurements}

Forward and reflected power waveforms were recorded at the front and rear end of a bidirectional coupler with the attenuations of 23 and $13 \mathrm{~dB}$, respectively. Further, in the case of reflected power waveform, pyramidal horn acted as the receiving antenna at the aperture, whereby the reflected beam passed through a ceramic power attenuation factor of $\frac{1}{\sqrt{\epsilon}} \approx \frac{1}{3}$ that has to be taken into account.

The actual power waveforms are evaluated from the detector voltage waveforms, according to

$$
\begin{gathered}
P_{F}(t)=P_{\text {peak }} \times \frac{V_{F P}(t)}{V_{F P \max }}, \\
P_{R}(t)=P_{\text {peak }} \times \frac{V_{R P}(t)}{10 \times V_{R P \max }} \sqrt{\epsilon}, \\
P_{T}(t)=P_{F}(t)-P_{R}(t),
\end{gathered}
$$

where $P_{F}, P_{R}$, and $P_{T}$ are the forward, reflected and transmitted power, respectively, $V_{F P}(t)$ and $V_{R P}(t)$ are measured forward and reflected voltage waveforms, respectively, and $V_{F P \max }$ and $V_{R P \max }$ are their maximum values.

Figure 3 shows the waveforms for the forward, reflected and transmitted power evaluated from the detector signals. From the figure it can be seen that the reflected power signal was measured to be $\leq 20 \%$ of forward power signal. Still, the reflected power waveform quantifies the contribution of the reflected wave in the total field amplitude at the aperture and in the sustaining the standing wave discharge between the

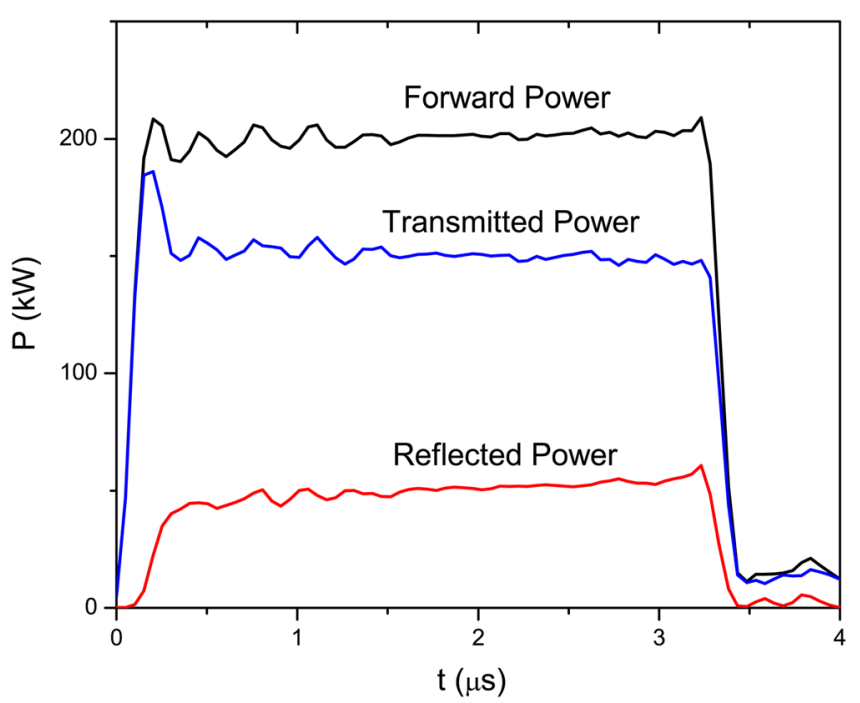

FIG. 3. (Color online) Forward, reflected and transmitted into plasma power waveforms from the (0-0) band of the Second Positive System of Nitrogen.

dielectric cover of the aperture and the plasma itself, in analogy to the cylindrical surface wave discharges.

\section{B. Ground-state population}

In weakly ionized plasmas (degree of ionization less than $1 \%$ ), ground state population $N_{n}$ can be approximated with the neutral particle density and is determined from the ideal gas equation

$$
N_{n}=\frac{p}{k_{B} T_{g}}
$$

where $p$ is the pressure in the static cell, $N_{n}$ is the groundstate number density (in $\mathrm{cm}^{-3}$ ) and $T_{g}$ is the gas kinetic temperature. Gas kinetic temperature is assumed equal to the rotational temperature of molecules in the discharge, if the molecular excited states are produced by direct electron excitation from the ground state. ${ }^{14}$ This assumption is used in our simplified kinetic model. In the Nitrogen Second Positive System the rotational spectrum consists of three branches: P, $\mathrm{Q}$, R. Both $\mathrm{P}$ and $\mathrm{R}$ branches are split into three subbranches and the $\mathrm{Q}$ branch into two due to the triplet splitting of the rotational quantum number, $J^{\prime}$. We have chosen to use $\Delta v=2$ vibrational sequence from the $\mathrm{R}_{2}$ branch of the $C^{3} \Pi_{u}$ $\rightarrow B^{3} \Pi_{g}$ band, since there is minimal self absorption and there is no interference from other atomic lines or molecular bands. Furthermore, we employed only lines with higher $J^{\prime}$ values, since they are less self absorbed than those at lower values.

Normalized intensity of a rotational line in the $R_{2}$ branch is given by

$$
I_{2, J^{\prime}}^{R}=S_{2, J^{\prime}}^{R} \frac{1}{Q} e^{-J^{\prime}\left(J^{\prime}+1\right) \frac{h c B_{x}}{k_{B} T_{r}}},
$$

where $S_{2, J^{\prime}}^{R}=\frac{\left(J^{\prime}+2\right)\left(J^{\prime}-2\right)}{J^{\prime}}$ is the line strength of the $R_{2}$ branch, $Q$ is the partition function, rotational constant $B_{x}=198.98 \mathrm{~m}^{-1}$ and $k_{B}$ is the Boltzmann constant. By calculating the slope of the Boltzmann distribution using Eq. (21), we were able to 
determine rotational temperature, $T_{r}=(720 \pm 70) \mathrm{K}$. The error reflects the uncertainty in measuring spectral line intensities. This result was confirmed by comparing experimentally obtained spectra with theoretically simulated synthetic spectra that is, presented in Fig. 4.

Using the measured gas temperature, we were able to apply Eq. (20) and calculate ground state number density. Knowing the chamber pressure, $p=11.8$ Torr, the ground state number density was evaluated at $N_{n}=(1.6 \pm 0.2) \times 10^{17} \mathrm{~cm}^{-3}$.

\section{Populations of vibrationally excited C states}

The time scale of the microwave pulses in the present case is long enough for the distribution of $N\left(C_{i}\right)$ states to be expressed by the vibrational temperature $T_{v}$ as a single statistical parameter by using the Eq. (7). Thus, it was necessary for further analysis to determine the value of the vibrational temperature.

In order to minimize the error due to the self-absorption, we have chosen to use $C^{3} \Pi_{u} \rightarrow B^{3} \Pi_{g}, \Delta v=2$ transition for determination of the vibrational temperature. By assuming that a Boltzmann factor is applicable to the limited number of the observed vibrational states we have introduced the proportionality equation

$$
I_{v^{\prime} v^{\prime \prime}} \approx A_{v^{\prime} v^{\prime \prime}} \nu_{v^{\prime} v^{\prime \prime}} e^{-\frac{G_{0}\left(v^{\prime}\right) h c}{k T_{v}}},
$$

where $A_{v^{\prime} v^{\prime \prime}}$ is the Frank-Condon factor for the transition between the upper $\left(v^{\prime}\right)$ and lower $\left(v^{\prime \prime}\right)$ state, ${ }^{15} \nu_{v^{\prime} v^{\prime \prime}}$ is the transition frequency, $G_{0}\left(v^{\prime}\right) h c$ is the energy of the vibrational level. It is defined as

$$
\begin{aligned}
G_{0}\left(v^{\prime}\right)= & \omega_{e}\left(v^{\prime}+\frac{1}{2}\right)-\omega_{e} x_{e}\left(v^{\prime}+\frac{1}{2}\right)^{2} \\
& +\omega_{e} y_{e}\left(v^{\prime}+\frac{1}{2}\right)^{3}+\omega_{e} z_{e}\left(v^{\prime}+\frac{1}{2}\right)^{4},
\end{aligned}
$$

where $T_{v}$ is the vibrational temperature. Using the Boltzmann plot, similar to the determination of rotational temperature,
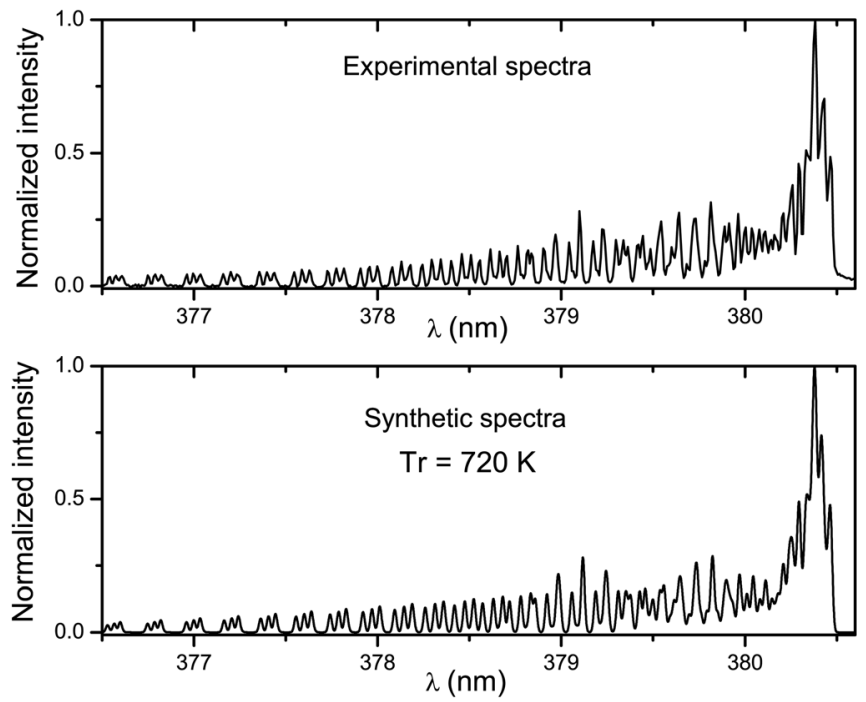

FIG. 4. Comparison between the experimental and synthetic spectra of Nitrogen Second Positive System. the vibrational temperature was found to be $T_{v}=$ $(3300 \pm 200) \mathrm{K}$.

Figure 5 shows vibrational distribution of $C^{3} \Pi_{u}$ state for the first three vibrational levels obtained from absolute intensities of the bands belonging to $\Delta v=2$ sequences. It fits very well with the Boltzmann distribution justifying the assumption of the $\mathrm{V}-\mathrm{V}$ equilibrium.

\section{Rate coefficients}

Rate of population of the vibrational level $C$ by the electron impact, $k_{e}$ depends strongly on the reduced electric field $E / N$ and vibrational temperature $T_{v}$. Space-average peak electric field waveform depends on average peak power density, $P_{\text {ave }}$. Assuming the transmission network impedance, $Z=50 \Omega$ we have

$$
E_{\text {ave }}=\sqrt{\Pi \times Z} \approx 478 \frac{V}{\mathrm{~cm}} .
$$

At the chamber pressure $p=11.8$ Torr and room temperature the averaged peak reduced field is therefore

$$
\left(\frac{E}{N}\right)_{\text {ave }}=126 T d .
$$

We conclude that the peak power of $210 \mathrm{~kW}$ at the waveform plateau corresponds to the average reduced electric field of $126 \mathrm{Td}$. Using data for $k_{e}$ from Ref. 16, we used a spline fit to obtain $k_{e}(E / N)$ as follows

$$
\begin{gathered}
k_{e}=10^{2.88 \ln (E / N)-23.98} \text { for }\left(\frac{E}{N}\right)<100 T d \\
k_{e}=10^{1.79 \ln (E / N)-18.93} \text { for }\left(\frac{E}{N}\right)>100 T d .
\end{gathered}
$$

where $(E / N)$ is given in $\mathrm{Td}$ and $k_{e}$ in $\mathrm{cm}^{3} / \mathrm{s}$.

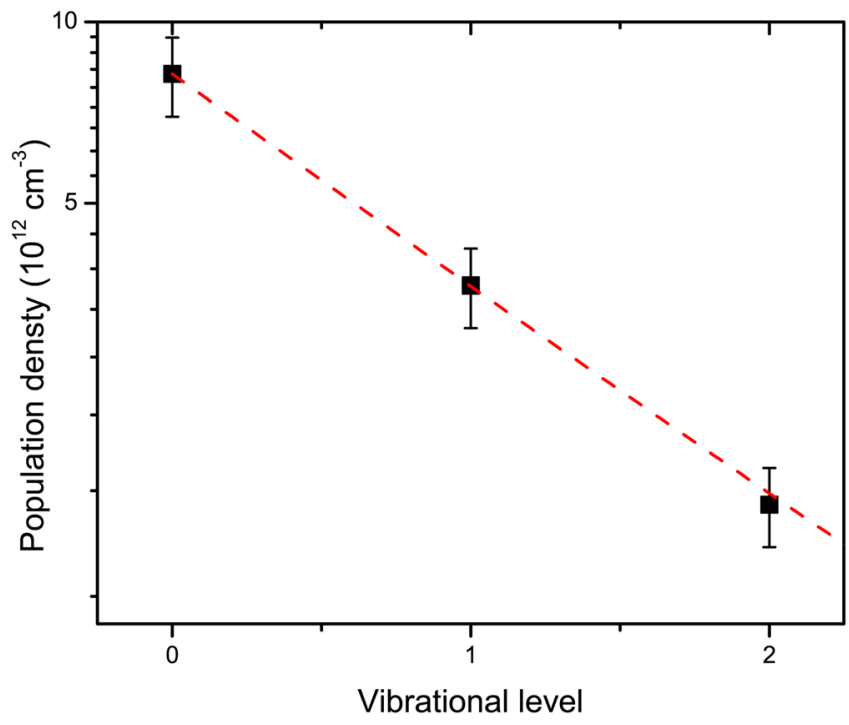

FIG. 5. (Color online) Vibrational population distribution of nitrogen $\mathrm{C}$ states measured from emission spectrum and estimated from Boltzmann distribution at $T_{v}=3300 \mathrm{~K}$. 
For simplicity, the effect of the relaxation of electron energy distribution function (EEDF) was neglected. In the initial $0.5 \mu$ s the EEDF is dominated by inelastic collisions, mostly by ionization, and differs substantially from the EEDF applied in the rest of the pulse, which is based on the full set of electron-molecule collisions. Small values of $N_{e}$ in the time interval of discharge build-up will make the effect of time dependent EEDF small. Still, evaluation of EEDF at the inception of the microwave discharge is certainly an important task for future work. The actual reduced electric field at the plasma can be calculated from the average power per unit area transported by microwave intensity,

$$
I \sim\left(\frac{E}{N}\right)^{2}
$$

and taking into account

$$
I=I_{F}-I_{R}
$$

where $I_{F}$ is the intensity of the forward (travelling) wave and $I_{R}$ is the intensity of the reflected wave. Thus,

$$
\left(\frac{E}{N}\right)^{2}(t)=\left(\frac{E}{N}\right)_{F}^{2}(t)-\left(\frac{E}{N}\right)_{R}^{2}(t)
$$

where $(E / N)_{F}(t)$ is reduced electric field associated with the forward wave and $(E / N)_{R}(t)$ is the reduced electric field associated with reflected wave. If we introduce the reflection coefficient

$$
\Gamma(t)=\sqrt{\frac{P_{R}(t)}{P_{F}(t)}}=\frac{\left(\frac{E}{N}\right)_{R}(t)}{\left(\frac{E}{N}\right)_{F}(t)},
$$

we derive

$$
\left(\frac{E}{N}\right)(t)=\left(\frac{E}{N}\right)_{F} \sqrt{1-\Gamma^{2}(t)}
$$

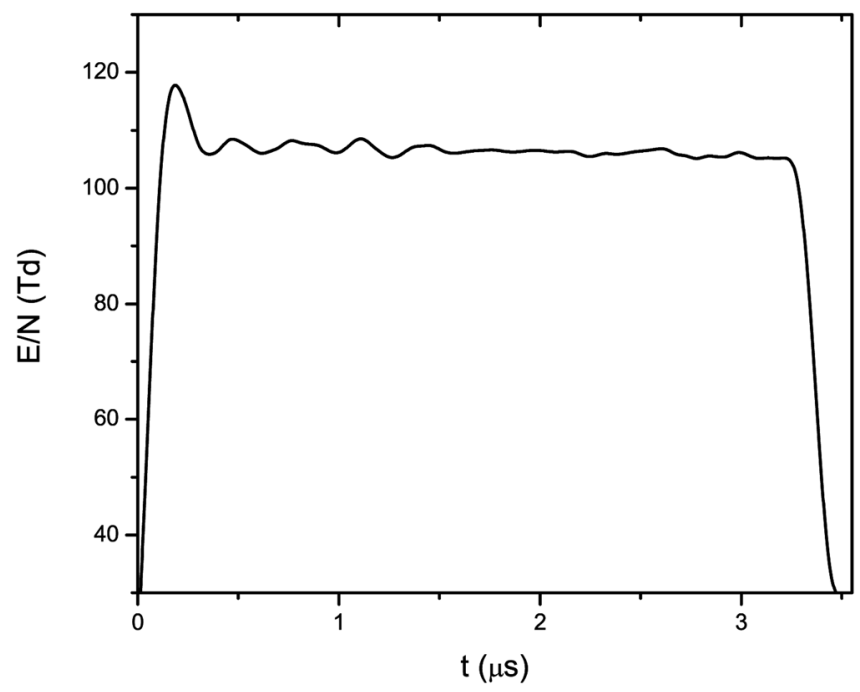

FIG. 6. Reduced electric field evaluated from the waveform of the $(0-0)$ band in the Second Positive System of Nitrogen.

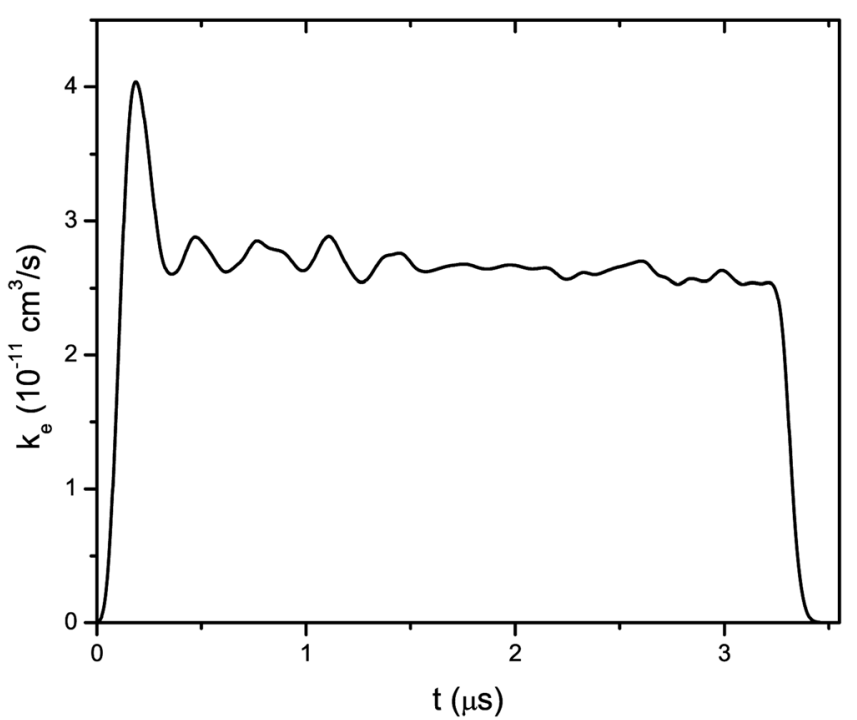

FIG. 7. Rate coefficient for excitation by electron impact evaluated from the waveform of the (0-0) band in the Second Positive System of Nitrogen.

The variation of the reduced electric field and rate coefficient for excitation by electron impact in the present case are given in Figs. 6 and 7, respectively.

It can be seen that the reduced electric field is almost constant for most of the pulse duration (see Fig. 6) allowing us to apply the rate coefficients for vibrational excitation by electron impact $k_{e}^{0, v}$ and $k_{e}^{v, 0}$, with a constant value. In the first approximation, all rate coefficients for vibrational excitation by electron impact were determined by employing the work of Hagelaar and Pitchford, ${ }^{17}$ assuming that for this particular case the EEDF was constant during the pulse and dependent solely on the self-consistent electrical field.

There are still no measured nor calculated total crosssections for vibrational excitation of the $C^{3} \Pi_{u}$ state available. In order to estimate rate coefficients, we have taken the advantage of the fact that the vibrational excitation rates of the nitrogen ground state have been reported. Therefore, we estimate $k_{e}^{0, v}$ and $k_{e}^{v, 0}$ by scaling the values of rate coefficients for the vibrational levels in $N_{2}\left(X_{1} \sum_{g}^{+}\right)$state. Results for the rate coefficients in the ground state are given in the Table I. The same concern arises in evaluation of rate coefficients for $\mathrm{V}-\mathrm{V}$ processes, $k_{v, v-1}^{0,1}$ and $k_{v-1, v}^{1,0}$. By applying the work of Capitelli and Dilonardo, ${ }^{18}$ we estimated these coefficients to be about $10^{-18} \mathrm{~cm}^{3} / \mathrm{s}$. The next step was to evaluate the rate constant for collisional deactivation. Quenching rate constant, $k_{c}$, is a function of density and vibrational quantum number. It is equal to $k_{c}=2.7 \times 10^{-11} \mathrm{~cm}^{3} / \mathrm{s}$ when the pressure in the cell is $p=11.8$ Torr. ${ }^{19}$

We should mention that in discharges containing air nitrogen oxide, NO, which is highly reactive could be produced in observable quantities. In this work, we evaluated the presence of nitric oxide by comparing the radiation from

TABLE I. Rate coefficients for the electron-impact vibrational excitation of the ground-state nitrogen.

\begin{tabular}{lcccc}
\hline \hline$v$ & 1 & 2 & 3 & 4 \\
\hline$k_{i g} \times 10^{-10}\left(\frac{\mathrm{cm}^{3}}{\mathrm{~s}}\right)$ & 41.6 & 23.8 & 16.2 & 9.37 \\
\hline \hline
\end{tabular}




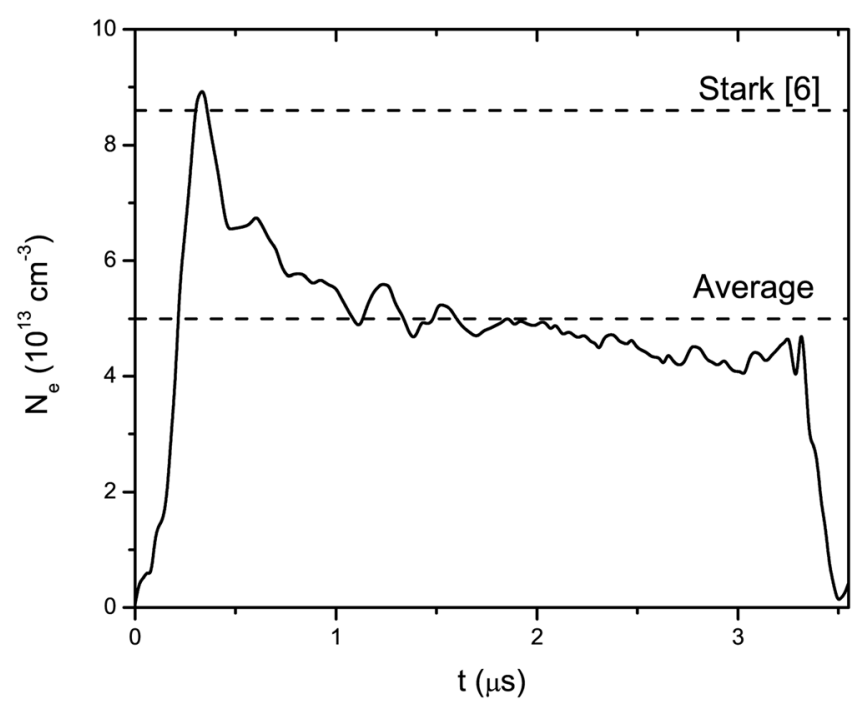

FIG. 8. Electron density evaluated numerically.

nitrogen and nitric oxide bands. We found that $\mathrm{NO} \gamma$ bands could be barely observed. Thus, we conclude that NO presence is negligible.

\section{E. Electron density}

Vibrational population of the $\mathrm{C}$ state depends on two main terms, vibrational excitation by electron impact within the $N_{2}\left(C^{3} \Pi_{u}\right)$ state, and vibration-vibration collisions within the same state, described in detail in the Kinetic Model Section. We estimated the contributions from both $e-\mathrm{V}$ and $\mathrm{V}-\mathrm{V}$ collisional processes by using rate coefficients for the nitrogen ground state in the absence of the proper coefficient rates for the $\mathrm{C}$ state. The terms due to the $e-\mathrm{V}$ processes are nine orders of magnitude smaller than any other term in the Eq. (6) and the terms due to the $\mathrm{V}-\mathrm{V}$ processes contribute even less. Rates for the vibrational excitation of the $N_{2}\left(C^{3} \Pi_{u}\right)$ state are even smaller than the ones for the ground state which justifies neglecting them. Thus, the last four terms in Eq. (6) are neglected in the numerical solution, as well as for the analytical approximation.

Electron density was first estimated by using Eq. (10) for the steady state analytic method. For the pressure of $p=11.8$ Torr, electron density was determined as $N_{e}=(4.9 \pm 0.6) \times 10^{13} \mathrm{~cm}^{-3}$. We assumed that the relative errors in pressure and $k_{e}$ measurements were no more than $5 \%$. The electron density waveform was then obtained numerically in two steps. First, we calculated the first derivative of the measured $\left[N_{2}\left(C_{v=0}\right)\right](t)$ state and then we used this value to determine electron density waveform from Eq. (6). The results are presented in Fig. 8. The average value of the electron density from the numerical method was calculated to be $(5.0 \pm 0.5) \times 10^{13} \mathrm{~cm}^{-3}$ over the interval $0.1-4.0 \mu \mathrm{s}$, and is indicated in the figure with a dashed horizontal line.

These values are lower than the results obtained using Stark broadening as in Ref. 6. There are two reasons for such a discrepancy. First, for the plasma conditions at hand, the deconvolution of the Stark width from the measured Voigt profile was limited by the instrumental resolution of the spectrometer. Second, the intensity-to-noise ratio of the $H_{\beta}$ line was rather low. Those uncertainties led the authors ${ }^{6}$ to estimate the spread of the electron density value obtained from Stark broadening of $H_{\beta}$ line of $(8 \pm 3) \times 10^{13} \mathrm{~cm}^{-3}$ which is in fair agreement with electron densities obtained in this paper.

\section{CONCLUSION}

A technique for evaluation of electron density has been developed and demonstrated. It is a non-intrusive method based on analysis of molecular bands of nitrogen $C^{3} \Pi_{u} \rightarrow$ $B^{3} \Pi_{g}$ second positive system. Both electronic and vibrational states were included in the study. A simple, but accurate kinetic model that calculates excitation rates of $N_{2}\left(C^{3} \Pi\right)$ system was employed. Resulting equations were solved numerically. This technique covers the gap between the Langmuir probe and Stark broadening measurements. The method was tested in the air surface plasma generated at the aperture of the horn antenna by a pulsed repetitive microwave beam. It has universal application in all pulse repetitive discharge plasmas. Optical emission spectroscopy was used to obtain the plasma parameters necessary for solving the model equations. It was possible to time resolve the electron density on sub microsecond scale. The process consists of two stages, unsteady state where rate constants are not fully defined and the steady state with all parameters completely described. Numerical and analytical solutions predict the similar time-average electron densities and their favourable comparison with electron densities obtained from Stark broadening technique indicates that the proposed method is reliable at electron densities bellow $10^{14} \mathrm{~cm}^{-3}$ and could be recommended as a convenient procedure for diagnostics of parameters in pulse-repetitive plasmas at atmospheric and near-atmospheric pressures.

${ }^{1}$ Y. P. Raizer, Gas Discharge Physics (Springer, Berlin, 1991).

${ }^{2}$ V. A. Godyak, R. B. Piejak, and B. M. Alexandrovich, Plasma Sources Sci. Technol. 11, 525 (2002).

${ }^{3}$ T. J. M. Boyd and J. J. Sanderson, The Physics of Plasmas (Cambridge University Press, Cambridge, United Kingdom, 2003).

${ }^{4}$ S. D. Popa, J. Phys. D: Appl. Phys. 29, 416 (1996).

${ }^{5}$ F. Cramarossa, G. Ferraro, and E. Molinari, J. Quant. Spectrosc. Radiat. Transf. 14, 419 (1974).

${ }^{6}$ R. J. Exton, R. J. Balla, G. C. Herring, S. Popović, and L. Vušković, AIAA Paper No. 2003-4181, "Volumetric Near-Field Microwave Plasma Generation" (2003).

${ }^{7}$ M. Ivković, S. Jovićević, and N. Konjević, Spectrochim. Acta, Part B 59, 591 (2004).

${ }^{8}$ K. V. Kozlov, H.-E. Wagner, R. Brandenburg, and P. Michel, J. Phys. D: Appl. Phys. 34, 3164 (2001).

${ }^{9}$ P. Paris, M. Aints, F. Valk, T. Plank, A. Haljaste, K. V. Kozlov, and H.-E. Wagner, J. Phys. D: Appl. Phys. 38, 3894 (2005).

${ }^{10}$ J. H. Kim, Y. H. Choi, and Y. S. Hwang, Phys. Plasmas 13, 093501 (2006).

${ }^{11}$ X. M. Zhu, Y. D. Pu, Z. G. Guo, and Y. K. Pu, Phys. Plasmas 13, 123501 (2006).

${ }^{12}$ S. V. Pancheshnuyi, S. M. Starikovskaia, and A. Yu. Starikovskii, J. Phys. D: Appl. Phys. 32, 2219 (1999).

${ }^{13}$ G. C. Herring and S. Popović, Appl. Phys. Lett. 92, 131501 (2008).

${ }^{14}$ D. J. Drake, Microwave Cavity Discharges in a Supersonic Flow (Lambert Academic Press, Saarbrücken, Germany, 2009).

${ }^{15}$ G. Hartmann and P. C. Johnson, J. Phys. B 11, 1597 (1978).

${ }^{16}$ J. Loureiro and C. M. Ferreira, J. Phys. D: Appl. Phys. 22, 67 (1989).

${ }^{17}$ G. J. M. Hagelaar and L. C. Pitchford, Plasma Sources Sci. Technol. 14, 722 (2005).

${ }^{18}$ M. Capitelli and M. Dilonardo, Rev. Phys. Appl. 13115 (1978).

${ }^{19}$ B. Brocklehurst and F. A. Downing, J. Chem. Phys. 46, 2976 (1967). 